\title{
LA INTERPRETACION AMBIENTAL COMO ESTRATEGIA EN EDUCACIÓN AMBIENTAL CON ESCOLARES DE BASICA PRIMARIA DEL MUNICIPIO DE LETICIA (AMAZONAS) EN LA RESERVA AGAPE
}

\author{
ENVIRONMENTAL INTERPRETATION AS A STRATEGY IN ENVIROMENTAL \\ EDUCATION WITH SCHOOLCHILDREN OF PRIMARY BASIC STUDIES AT THE \\ AGAPE RESERVE OF THE MUNICIPALITY OF LETICIA (AMAZONAS)
}

\author{
Fanny Matilde Pinzón Candelario \\ Licenciada en Biología \\ Especialista en Ed. Ambiental \\ Magister en Ing. Ambiental \\ Universidad Nacional Abierta y a Distancia \\ ORCID: https://orcid.org/0000-0002-2082-2971
}

Correo: fanny.pinzon@unad.edu.co

Citación: Pinzón, F. (2021). La interpretación ambiental como estrategia en educación ambiental con escolares de básica primaria del Municipio de LETICIA (AMAZONAS) en la reserva AGAPE. Working Papers ECAPMA, 1, 27 - 43. DOI: https://doi.org/10.22490/ECAPMA.4232

\section{RESUMEN}

El presente documento es el resultado de un trabajo, cuyo objetivo era sensibilizar mediante la estrategia de interpretación ambiental, a estudiantes de básica primaria de los grados 4to y 5to del Municipio de Leticia (Amazonas), sobre el concepto de ambiente y la importancia de conservarlo. Se utilizó como metodología la Investigación acción participativa, mediante el desarrollo de una estrategia de Educación ambiental, como es la interpretación ambiental en senderos ya establecidos en la Reserva Ágape, ubicada en el Km
10,5 Vía Tarapacá, zona rural de la ciudad de Leticia, Amazonas. Uno de los hallazgos es que el conocimiento que tienen los niños sobre su ambiente es mínimo.

Por medio de la estrategia de interpretación ambiental que tiene sus bases en un guion elaborado previamente para cada sendero, que incluye contenido conceptual y metafórico, se logró sensibilizar a los niños; la actividad incluía experiencias sensoriales, de juego, actividades de preservación, conservación, que permitieran fortalecer sus conocimientos, permitiendo la 
construcción de valores ambientales buscando generar nuevos patrones de conducta frente al ambiente $y$ modificar patrones de comportamiento.

Se concluyó que este tipo de actividades de interpretación ambiental, que incluye la participación de todos los sentidos en espacios abiertos accesibles, permite generar la curiosidad de los niños por conocer y explorar, más allá de su conocimiento formal de aula. Se evidencia que no es suficiente conocer sobre la problemática ambiental si esta, no se conoce en la realidad; más allá del conocimiento que se puede lograr en las aulas convencionales.

Palabras Clave: Interpretación ambiental; espacio; abióticos; bióticos; antrópicos

\section{ABSTRACT}

This document is the result of a work, whose objective was to sensitize students of primary school in grades 4 and 5 of the municipality of Leticia (Amazonas) through the environmental interpretation strategy, on the concept of the environment and the importance of preserving it.

\section{INTRODUCCIÓN}

El planeta en la actualidad enfrenta una problemática ambiental que viene generando cambios sustanciales como perdida de vegetación, desertificación, cambios en el clima global del planeta,
Through the strategy of environmental interpretation that has its basis in a script prepared previously for each path, which included conceptual and metaphorical content, children were sensitized; the activity included sensory experiences, of play, activities of preservation, conservation, that would allow to strengthen their knowledge, allowing the construction of environmental values seeking to generate new patterns of behavior in the face of the environment and modify behavior patterns.

It was concluded that this type of environmental interpretation activities, which includes the participation of all senses in accessible open spaces, allows to generate the curiosity of children to know and explore, beyond their formal classroom knowledge. It is evident that it is not enough to know about environmental problems if it is not known in reality; beyond the knowledge that can be achieved in conventional classrooms.

Keywords:

Environmental interpretation; space; biotics; biotics; anthropic

Iluvias acidas, perdida de especies de fauna y flora, debido a las actividades inconscientes de la humanidad. Es por esto por lo que se hace prioritario educar en educación ambiental y promover valores ambientales, que con 
el tiempo han ido desapareciendo, precisamente por el alejamiento que los humanos han tenido frente a la naturaleza, ya que no se sienten parte de ella sino dueños de ella.

En este sentido hoy más que nunca cualquier esfuerzo que se haga en relación con fomentar y sensibilizar a las personas frente al ambiente no sobra, por el contrario, es de vital importancia si él interés es perpetuar la raza humana. Se debe entonces educar para buscar cambios de conducta $y$ reorientar los comportamientos poco amigables con el ambiente, en función de formar ciudadanos equilibrados con su entorno.

Estas problemáticas ambientales nos hacen reflexionar sobre ¿qué se ha dejado de hacer para llegar a la situación actual?, con las graves consecuencias que se observa diariamente y ¿qué se debe hacer para generar un cambio? Casi siempre la respuesta a esta pregunta está orientada a la educación y más exactamente a la educación ambiental y la construcción de valores ambientales que se fueron perdiendo con el tiempo.

Por lo anterior la pérdida de valores ambientales y el no seguir inculcándolos en la sociedad es lo que viene generando la destrucción del ambiente desde todos sus componentes, abióticos, bióticos y antrópico, ya que estos deben funcionar en un sistema equilibrado que permita el desarrollo sostenible de la sociedad y propenda por el mejoramiento de la calidad de vida.

Así pues, los valores ambientales son actos, acciones y comportamientos positivos del hombre frente al ambiente, valorando su entorno, respetándolo, conservándolo y haciendo un uso consciente $y$ responsable del mismo.

Como todos los demás valores, estos se van construyendo e interiorizando desde la infancia en los procesos que tiene el niño inicialmente con sus padres, familia y entorno inmediato, posteriormente son afianzados en la escuela. Sin embargo, si esto realmente se diera no se estarían presentando las grandes catástrofes ambientales que se están dando. Lo que significa que se deben hacer esfuerzos mayores $y$ utilizar estrategias que permitan realmente generar esta apropiación de valores ambientales $y$ que se mantengan en la adultez, para que éstos sean los nuevos referentes para enseñar a las generaciones futuras en un ciclo continúo basado en el ejemplo.

La educación ambiental entonces se incorpora como una de las principales herramientas para orientar estos cambios de comportamiento, pues busca que los actores participantes reflexionen sobre su relación con el medio ambiente y si este es el más adecuado. Como señalan Medraño, 
Goñi y Palacios (1999), esto debería poder brindar una referencia para el desempeño ideal humano para que no solo puedan evaluar el comportamiento de los demás, sino también su comportamiento en el entorno.

El problema actual no es sólo la pérdida de especies o el cambio climático, sino también de mayor alcance, es la pérdida de la naturaleza humana, en este caso el hombre y la naturaleza han perdido por completo su relación. Como dijo Stutzin (1984), los humanos se están convirtiendo en un enemigo de la naturaleza, enfrentando una guerra cada vez más intensa y feroz contra ella.

Con el mayor deseo de conquistar el mundo, la humanidad impone la tecnosfera sobre la biosfera. El resultado es que, se dé un ataque a la propia naturaleza humana. Si este fenómeno no se detiene, eventualmente traerá terribles consecuencias para las nuevas generaciones. Dadas estas situaciones se debe fomentar un cambio en el comportamiento de la nueva generación y promover el derecho a que la naturaleza debe existir con la raza humana. El ser humano es otra especie en la biosfera, por lo que no es de su propiedad, como cualquier otra especie, es parte del ciclo de vida, por lo que su derecho a la existencia es el mismo que el de otras especies (Rodriguez,2014). Los recursos de la biosfera existen para ser compartidos entre todas las especies, manteniendo así un complejo ciclo de equilibrio.

Es importante resaltar que el universo siempre busca la entropía, lo explica la segunda ley de la termodinámica que sostiene que todos los procesos que ocurren en el universo se realizan de manera que siempre aumenta el desorden y por tanto la entropía; sin embargo, los organismos son altamente organizados lo que sería aparentemente una contradicción a esta Ley, pero no es así, la explicación radica en que todos los organismos vivos, no importa si son unicelulares o pluricelulares, extraen energía del ambiente en donde se desarrollan, para aumentar y mantener su compleja organización. Por esta razón en los seres vivos disminuye la entropía, pero esa disminución de la entropía se mantiene aumentando la entropía a su alrededor, por lo que si los humanos como organismos pertenecientes de un sistema mayor, consumen más energía de la requerida en termino de uso de los recursos naturales renovables, estaría generando una entropía mayor en su entorno. (Gato,2019).

En este punto es donde la problemática de los valores ambientales salta a la palestra, ya que es evidente que este es el origen del problema ecológico y ambiental debido a los nuevos referentes valorativos que se manejan en la actualidad de consumismo. Por lo 
que se debe enfatizar que el respeto por la naturaleza no puede ser solo de los ambientalistas, sino que debe ser un valor ambiental formado para toda la población humana a través del ejemplo de la familia y en los procesos educativos iniciales del niño y reforzado en todo el crecimiento personal del individuo.

Al enfrentar la complejidad de la construcción de los conceptos sobre la naturaleza y como se da este proceso en el aula de clase, González (2007), hace referencia de la necesidad de incorporar procesos de alfabetización ambiental en todos los niveles, siendo tarea de todos y no solo de los docentes de ciencias naturales, sino que debe ser una construcción desde todas las áreas del conocimiento.

Por tanto, la educación ambiental tiene una alta relevancia, pero hay que cambiar el método de enseñanza y el método de desarrollo del proceso educativo, Acosta (2006) propone un innovador programa de educación ambiental que ayuda a formar personas que comprenden sus realidades sociales, históricas y culturales y tiene como objetivo mantener la calidad de vida.

En cuanto a los procedimientos de educación ambiental, se enfatizan las estrategias participativas en diferentes situaciones, por lo que Melendro (2008) propone que las estrategias para mejorar el proceso de enseñanza de la educación ambiental deben ser participativas. En el contexto de la comunidad, Tréllez (2002) propuso un método aplicable al proceso de educación ambiental probado en la experiencia de educación ambiental comunitaria de los países latinoamericanos, y utiliza técnicas participativas como los métodos situacionales escolares o de escenarios de la escuela.

Por otro lado, Molano (2004) utiliza métodos típicos del desarrollo rural participativo para integrar los conceptos y elementos prácticos de la educación ambiental para diagnosticar problemas y conflictos ambientales derivados de los sistemas de producción agrícola, e integrarlos. Asociado a grado. Conciencia ambiental, encontró que las herramientas utilizadas pueden mejorar la calidad de vida dentro de la comunidad, y reevaluar la relación entre los individuos y la comunidad, sus entornos naturales, naturales $y$ sociales, sus sistemas de producción y sus estilos de vida. A su vez, Sánchez (2009) integró la educación ambiental con la cultura comunitaria a través del juego. También se incluye la experiencia de Ángel Pérez (2011), utilizando la investigación acción (IA) como estrategia de enseñanza, exponiendo las actividades docentes de la ecología y la hidrología, y dejando aportes conceptuales y métodos para quienes participan en el desarrollo de 
proyectos. Por otro lado, según Trelles (2012), el entorno comunitario necesita herramientas, técnicas y métodos de formación para cultivar la acción y el pensamiento creativo, la renovación de las ideas y la imaginación, la dirección del cambio, la profundización de la conciencia del entorno de la vida cotidiana de la población, la participación y la acción son los elementos básicos de la educación ambiental comunitaria. Independientemente de la población utilizada o de la estrategia adoptada, el proceso de educación ambiental debe responder al desafío de educar sobre el ambiente o como señala Cánovas (2002), mediante la formación de relaciones sociales para enfrentar las complejas e interactivas relaciones sociales y naturales propendiendo, por la formación del pensamiento crítico, creativo y prospectivo, Novo (2009).

Por lo anterior el presente estudio busca contribuir en los procesos de educación ambiental de niños de básica primaria de una Institución educativa privada de la zona urbana del Municipio de Leticia (Amazonas), mediante estrategias que fomenten la formación de valores ambientales en la población escolar. El trabajo se desarrolla con apoyo de los docentes de los diferentes grados que participaron en las actividades.

\section{METODOLOGÍA}

Este estudio se realiza a través de la investigación-acción participativa, que es un método de investigación sociopsicológico basado en los siguientes elementos clave: múltiples sujetos participan juntos para desarrollar nuevos conocimientos. En otras palabras, la construcción de nuevos conocimientos se genera a partir de las acciones reflexivas de personas $y$ comunidades.

La IAP es un método de intervención en temas sociales, con el objetivo de hacer que el conocimiento generado por la investigación sea útil para la transformación social (Balcazar, 2003). Con este enfoque, esperamos que el desarrollo de las medidas de investigación e intervención se enfoquen en la participación de las personas que conforman la comunidad para la investigación e intervención, pues se entiende que la propia comunidad es la responsable de definir y orientar sus propias necesidades, conflictos y soluciones.

Para el caso del proyecto, se aplicó la estrategia de interpretación ambiental, mediante recorridos guiados en una Reserva Forestal que tiene como nombre Ágape, ubicada en el Km 10,5 vía Tarapacá en la zona rural del Municipio de Leticia (Amazonas). Ver imagen No 1. 


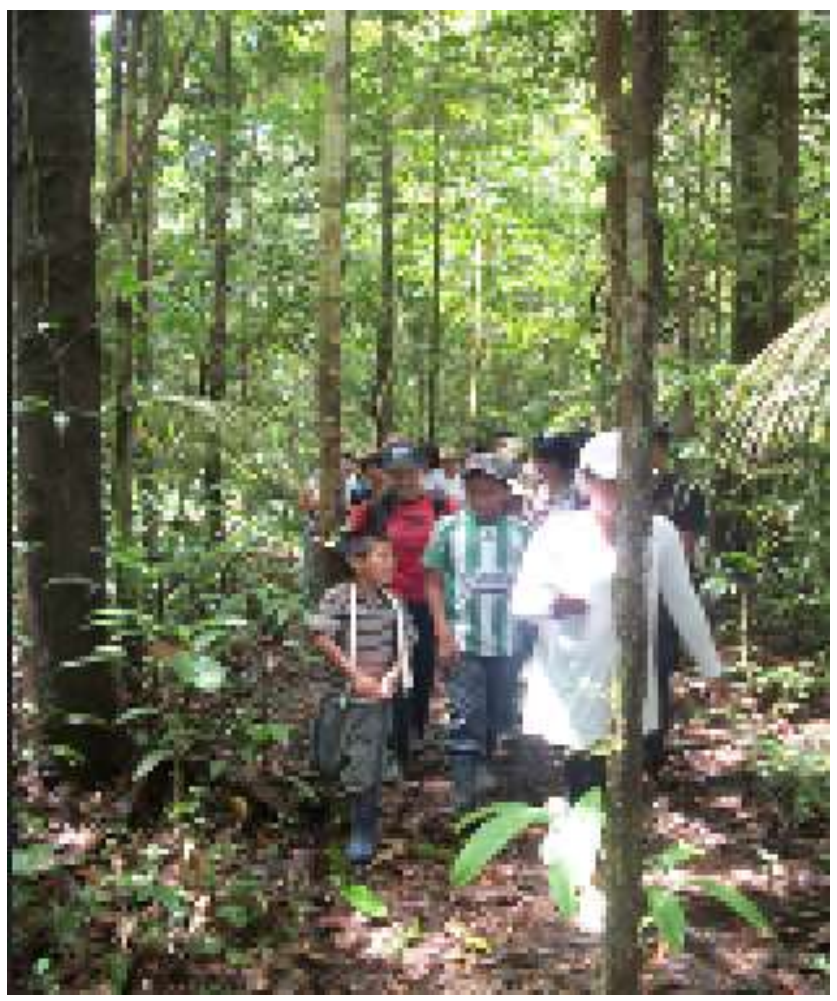

Imagen No 1. Recorrido de interpretación por los Senderos de la Reserva Agape. Fuente: autora.

Para la recolección de la percepción y conocimientos previos que traían los niños, se utilizó los métodos de investigación visual (dibujo). Este método es muy útil a la hora de trabajar con niños pequeños y permite estimular y motivar la participación, ya que crea un ambiente relajado y divertido para la investigación. Como es una actividad que realizan normalmente en el colegio - la casa, se sienten cómodos en desarrollarla y en ella pueden plasmar lo que quieran. Esta técnica del dibujo es una forma de expresión personal y una forma natural de comunicación por lo que se convierte en un método para que los niños representen las construcciones nuevas de sus conceptos, así que para este tipo de investigaciones de educación ambiental son muy valiosas, ya que permiten evaluar las representaciones gráficas, pasando de datos cualitativos a cuantitativos, lo que genera datos de fácil análisis.

\section{Participantes}

Se contó con la participación de 37 estudiantes de grado cuarto y quinto de primaria, del Colegio Cristo Rey del Municipio de Leticia- Amazonas que son habitantes del casco urbano de Leticia, 
con edades que oscilaban entre 12 y 15 años.

\section{Diseño Metodológico}

Para la etapa inicial de diagnóstico, se les solicitó a los niños participantes que realizaran un dibujo o esquema en el que representaran el concepto de ambiente. Esto permitiría conocer el conocimiento que tenían los niños antes de realizar el recorrido de interpretación ambiental por la reserva y ayuda a evaluar si el libreto establecido para el recorrido junto con las actividades propuestas en cada estación si lograba cambiar el concepto que los niños ya traían.

En un segundo momento, se realizó un recorrido de interpretación ambiental de 2 horas por un sedero ya establecido en una zona de bosque secundario y primario, dentro de la microcuenca de la quebrada la Arenosa, este sendero constaba de 10 estaciones, en su recorrido se iban mostrando especies de plantas nativas, fauna encontrada en el camino y se hablaba sobre la problemática que enfrenta el ambiente, se planteaban además actividades sensoriales donde los niños participaban activamente, basados en la hipótesis que todas las funciones sensoriales constituyen la estructura que permite a los humanos a tener contacto con el exterior e interiorizar al mismo tiempo sus experiencias, dándose procesos de aprendizaje significativo que permiten la construcción de nuevos conceptos.

Por otro lado, se puso a prueba el libreto diseñado para el recorrido de interpretación ambiental y las actividades organizadas previamente (abracemos un árbol, siente la energía, entre otros), en donde se abordaron conceptos complejos asociados a los recursos naturales (agua, aire, suelo, flora y fauna) como la fotosíntesis, los ciclos biogeoquímicos entre otros. Con el recorrido se buscaba que los niños ampliaran su concepto de ambiente y permitirles relacionarse con el entorno de una manera sensorial con la vista, el tacto, el gusto, el oído y el olfato, permitiendo así relacionarse con la selva y sentirse parte de ella.

Finalmente, después del recorrido debían realizar otro dibujo y volver a plasmar el concepto de ambiente.

\section{DISCUSIÓN}

\section{Primer momento:}

Los dibujos como estrategia para evaluar el concepto ambiente:

Los niños, aunque viven en el casco urbano de Leticia Amazonas, no mostraron mucho conocimiento sobre el ambiente de la región amazónica y su selva, algunos plasmaron en sus dibujos el esquema general cuando se representa un paisaje que incluye las montañas, el sol, una casa y un río, con un árbol. Por lo que no tuvieron en cuenta su entorno más cercano, y el 
ambiente en el que están inmersos en la zona Amazónica donde no se ven montañas, todo es netamente plano. (Ver Imagen No 2)

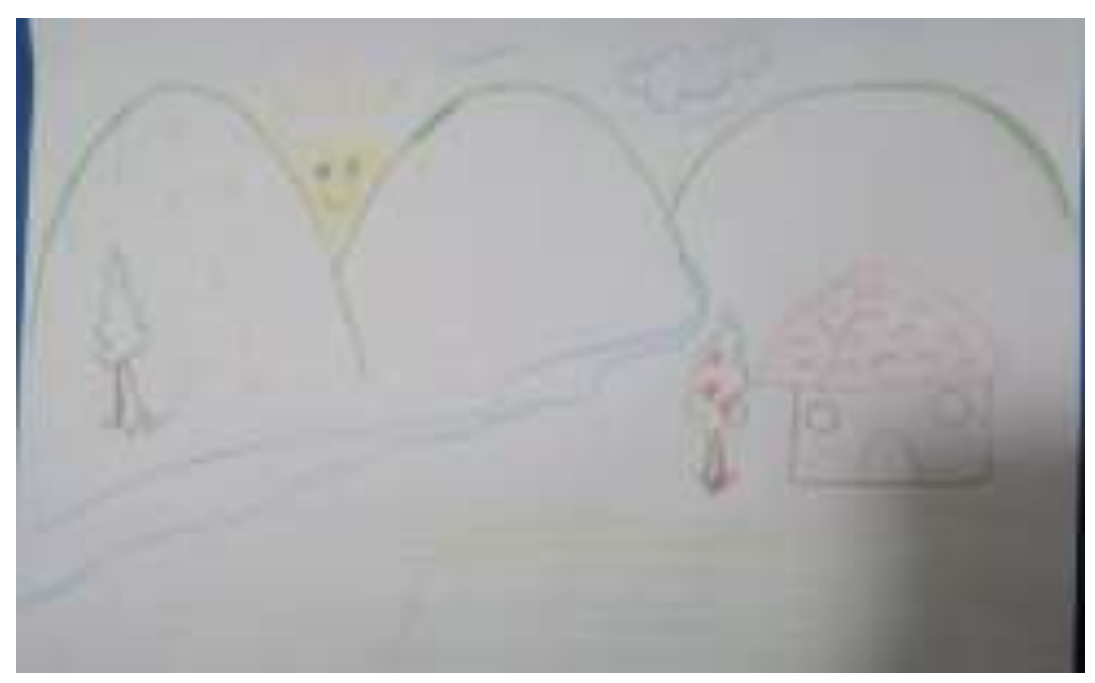

Imagen No 2: Representación gráfica de un participante momento 1. Fuente: autora con el permiso de la Fundación Amazonía Eware.

Algunos elaboraron dibujos del entorno de la ciudad y de sus viviendas, en donde no se veía reflejado, aspectos ecológicos, a pesar de que la ciudad está al borde del río Amazonas, muy pocos niños dibujaron el río y los que lo hicieron lo representaron de manera central y de tamaño grande. Recordemos que Leticia es la Capital del Departamento del Amazonas y está asentada al borde del río Amazonas, el río más caudaloso del mundo y que tiene una gran envergadura, por lo que se esperaría que formara parte de la mayoría de los dibujos de los niños.

Como el concepto que se estaba evaluando era el concepto de ambiente, se encontró que en algunos dibujos los niños solo ilustraron el entorno, pero no incluyeron figuras humanas o dibujos de ellos mismos, lo que nos hace pensar que ellos no se sienten parte del ambiente.

Para evaluar los dibujos se utilizó una estrategia de relaciones encontradas en los dibujos teniendo en cuenta el concepto de ambiente holístico en donde se dan relaciones bióticas, abióticas y antrópicas en un lugar y tiempo determinado. Para ello se estableció una matriz de relaciones así:

B-B: Relación biótica - biótica

A-B: Relación abiótica - biótica

A-A: Abiótica - abiótica

H-B: Antrópica - Biótica

H-A: Antrópica- abiótica

$\mathrm{H}-\mathrm{H}$ : Antrópica - Antrópica 
A-B-H Abiótica - Biótica- Antrópica

Cada dibujo se evaluó de acuerdo con el número de relaciones presentes en cada uno, con el fin de convertir esta información cualitativa en cuantitativa. Este es un ejemplo de dibujo (Ver imagen No 3).

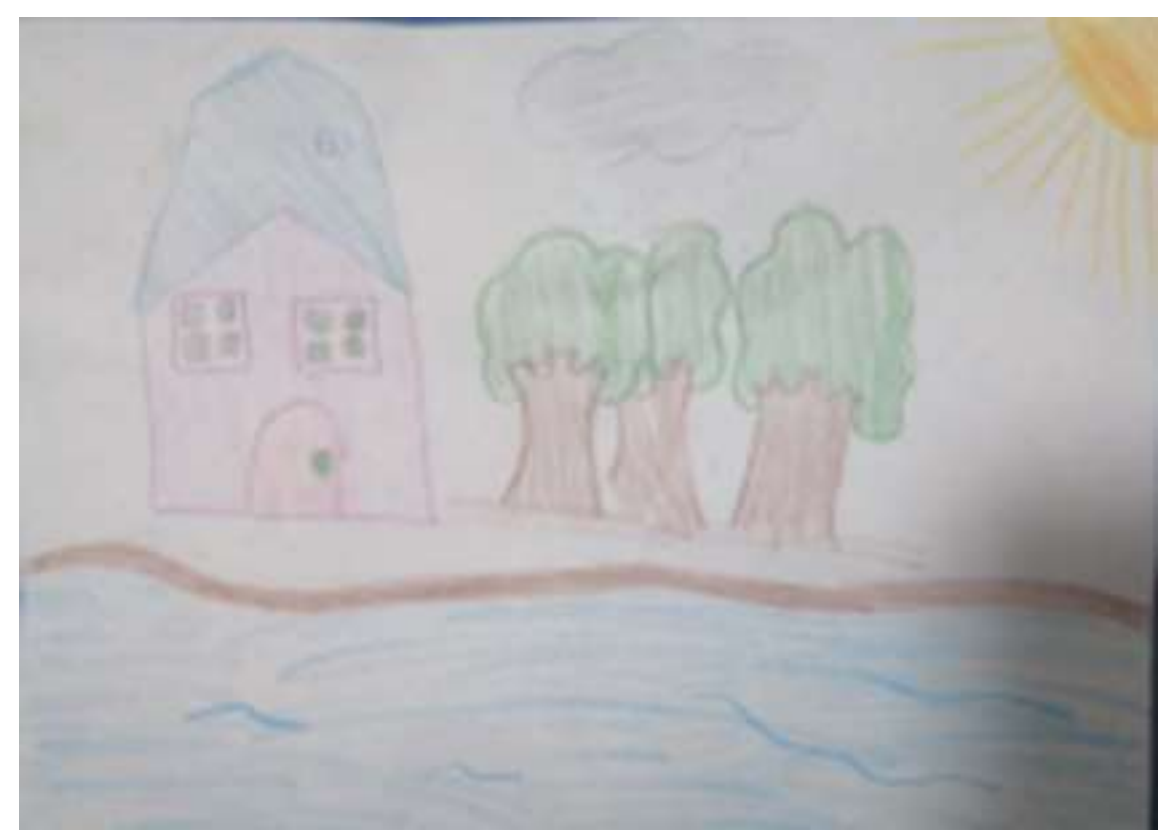

Imagen No 3. Dibujo de un participante en el momento 1. Fuente: autora con el permiso de la Fundación Amazonía Eware.

En la tabla No 1 se presenta la evaluación del dibujo presentado.

Tabla No 1. Resultado de evaluación de la representación realizada por el participante.

\begin{tabular}{lc}
\hline Relaciones & $\begin{array}{l}\text { \# de } \\
\text { relaciones } \\
\text { encontradas }\end{array}$ \\
\hline B-B: Relación biótica - biótica & 1 \\
\hline A-B: Relación abiótica biótica & 2 \\
\hline A-A: Abiótica - abiótica & 1 \\
\hline H-B: Antrópica - Biótica & 1 \\
\hline H-A: Antrópica- abiótica & 1 \\
\hline H-H: Antrópica - Antrópica & 0 \\
\hline A-B-H Abiótica - Biótica - Antrópica & 1 \\
\hline
\end{tabular}

Fuente: autora. 
En la gráfica No 1 encontramos los resultados de los 37 niños participantes en el momento 1:

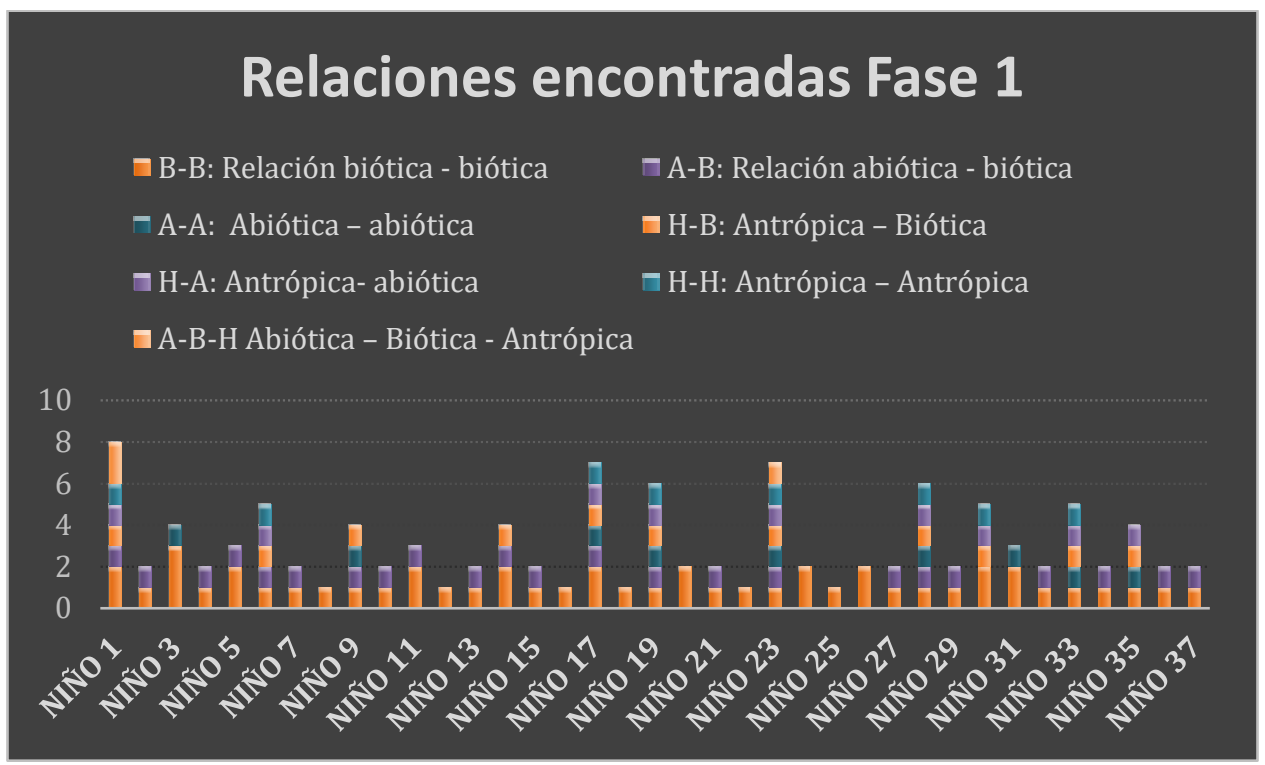

Gráfico No 1. Relaciones encontradas en los dibujos de los niños momento 1. Fuente: autora.

En la gráfica se puede evidenciar que el $60 \%$ de los niños solo plasmo entre 1 y 2 relaciones en su dibujo. El $27 \%$ entre 3 y 5 relaciones y el 13,5 entre 6 a 8 relaciones en los dibujos. Solo 2 niños representaron una relación Biótica-abiótica- antrópica, lo que equivale al $5,4 \%$ de todos los niños. Estos datos son muy bajos para el grupo muestra, teniendo en cuenta que viven en Leticia Amazonas un Municipio ubicado en plena Selva Amazónica.

Como estrategia para identificar el conocimiento espacial, al observar las representaciones dibujadas por los niños, aunque el entorno representado sea el mismo, es fácil notar que los elementos de cada dibujo son únicos y personalizados. No hay dos imágenes iguales, lo que puede confirmar Holahan (2005), quien mencionó que, para todos, un mismo espacio tiene diferentes significados según las actividades realizadas (trabajo, estudio, entretenimiento, etc.) Si se considera que la mayoría de los estudiantes tuvo una representación baja de los aspectos ambientales básicos en el primer dibujo (Fase 1), entonces se deben tomar acciones en el aula para adaptar a los individuos al espacio habitable correspondiente a su área de vida. (Martín, 1997) señaló en Caballero (2002) que la evidencia empírica muestra que la familiaridad con el entorno y la ubicación (urbana o 
rural) determina el nivel de representación espacial personal.

Caballero (2002) señaló que el vínculo emocional con el entorno afectará la expresión espacial que constituye y las actividades que se desarrollan en él. Esta afirmación es crucial y se confirma en los dibujos elaborados por los niños ya que ciertos procesos (como la deforestación, la contaminación de ríos, la caza de especies en peligro de extinción y el tráfico de animales) no se consideraron inicialmente un problema - amenaza a la biodiversidad de la zona.

Esto puede deberse a la limitada conexión emocional de los estudiantes con el entorno circundante 0 su desconocimiento de la riqueza a nivel ecológico; de manera similar, las familias de algunos estudiantes tienen fincas en el área, donde no hay una gestión ambiental sostenible, y por ende ellos no pueden visualizar claramente los daños o afectaciones que se tienen en la zona hasta que se les aborda en el recorrido del sendero de interpretación.

\section{Segundo momento:}

Algo diferente sucedió en los dibujos realizados luego de realizar el recorrido del sendero de interpretación, lo que permite inferir que el nivel inicial de representación de la zona inicialmente fue muy bajo, lo que puede deberse a una falta de comprensión de esta. Las imágenes plasmadas carecen de representaciones de fuentes de agua ricas en la zona y no evidencia la presencia de animales y plantas. En los esquemas representados en la fase 2 por parte de los niños, se han visto cambios significativos en los dibujos y se han establecido más relaciones Bióticas - abióticas - antrópicas, lo que sugiere que los niños han visto el concepto de medio ambiente desde una perspectiva más integral (holística), que era el objetivo de esta actividad. (Ver imagen No 4)

Por otro lado, algunos han incorporado en sus dibujos mensajes sobre el cuidado a la naturaleza, lo que implica una elaboración más elevada en la conceptualización, ya que se empieza a vislumbrar la inclusión de valores ambientales. 


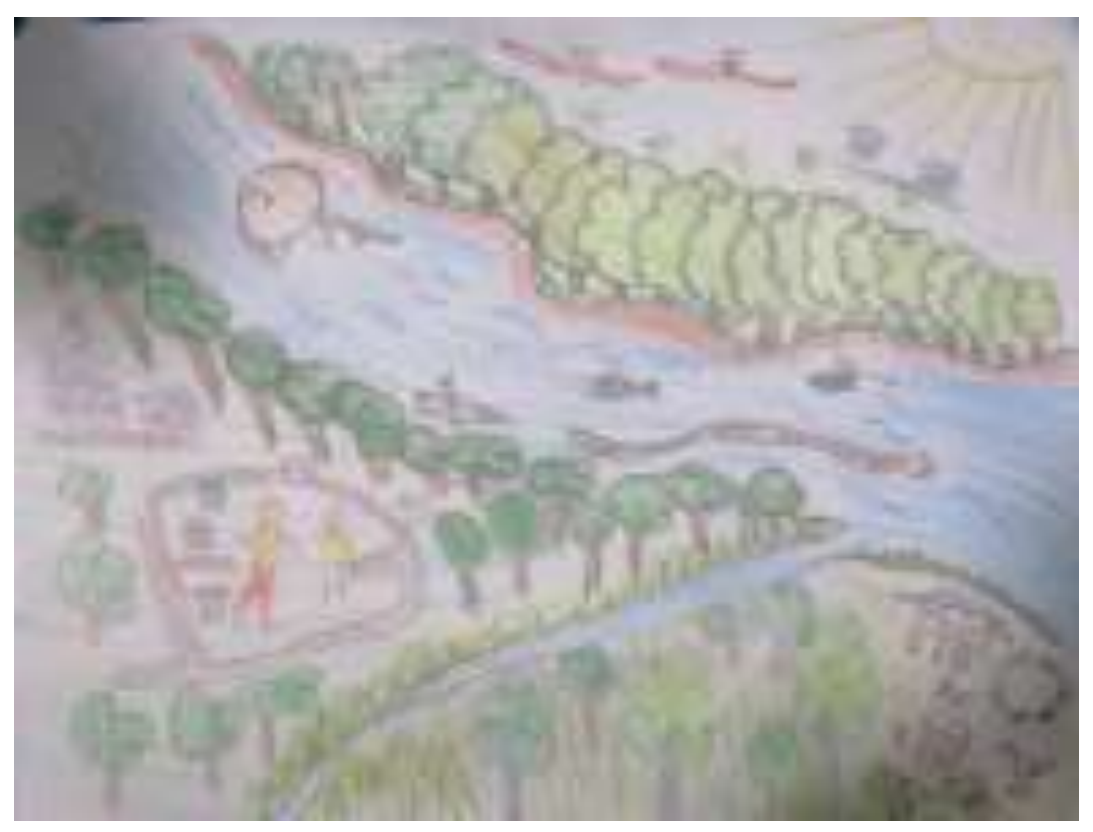

Imagen No 4. Dibujo de un participante en la Fase 2. Fuente: autora con el permiso de la Fundación Amazonía Eware.

A continuación, en la Tabla No 2 se encuentra la evaluación obtenida del anterior dibujo.

Tabla No 2. Resultado de evaluación de la representación realizada por el participante.

\begin{tabular}{lc}
\hline Relaciones & $\begin{array}{l}\text { \# de relaciones } \\
\text { encontradas }\end{array}$ \\
\hline $\begin{array}{l}\text { B-B: Relación biótica - } \\
\text { biótica }\end{array}$ & 3 \\
\hline $\begin{array}{l}\text { A-B: Relación abiótica - } \\
\text { biótica }\end{array}$ & 6 \\
\hline A-A: Abiótica - abiótica & 3 \\
\hline H-B: Antrópica - Biótica & 4 \\
\hline H-A: Antrópica- abiótica & 1 \\
\hline H-H: Antrópica - Antrópica & 2 \\
\hline $\begin{array}{l}\text { A-B-H Abiótica - Biótica - } \\
\text { Antrópica }\end{array}$ & 2 \\
\hline
\end{tabular}

Fuente: autora. 
En el gráfico No 2 se puede evidenciar los datos obtenidos de las relaciones plasmadas por los niños después del Sendero de interpretación.

A diferencia de la fase anterior los niños lograron plasmar muchas más relaciones en sus dibujos, el estudiante que menos relaciones plasmo represento 11 relaciones y el que más relaciones plasmo presento
21. El $21,62 \%$ de los niños represento entre 11 a 13 relaciones, el 37,8 representó entre 14 a 16 relaciones en sus dibujos, el 40, $5 \%$ incluyeron entre 17 a 21 relaciones en sus dibujos. Lo que significa que se tuvo un aumento significativo en las relaciones plasmadas por los niños de la Fase 1 y 2 , de más del $200 \%$.

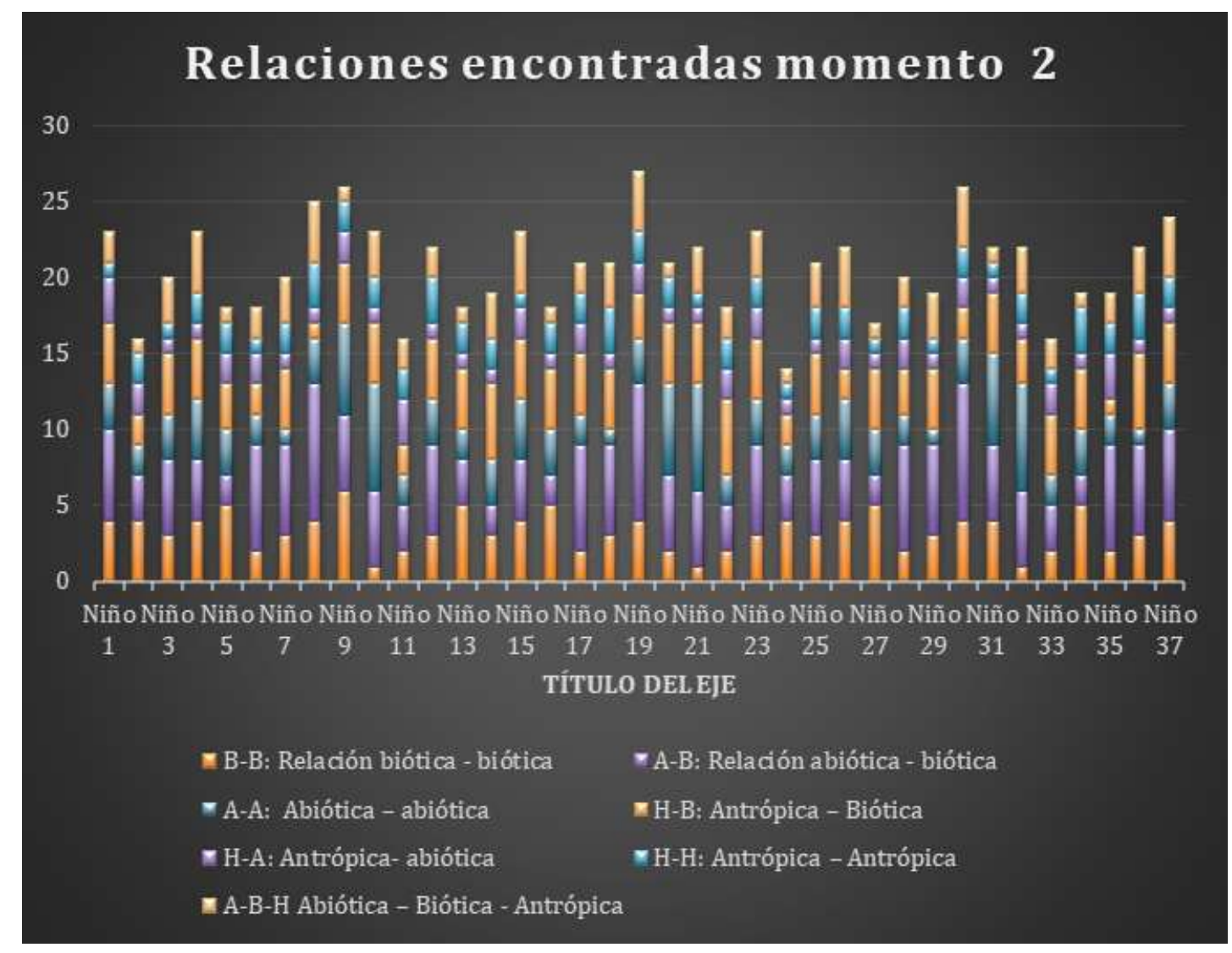

Gráfico No 2. Relaciones encontradas en los dibujos de los niños Momento 2. Fuente: autora.

Para autores como (Hart \& Moore, 1973) en Caballero (2002), no solo es importante el conocimiento espacial de la zona, sino también la relación interactiva que establecen los actores con ella, su uso y la sensación que les da o les provoca. Estos elementos se convierten en la base necesaria para el 
diseño de cualquier plan de educación ambiental, y los resultados obtenidos en el proceso de trabajo con los estudiantes dan cuenta que con un plan bien estructurado de interpretación y sensibilización del ambiente se pueden tener logros importantes en la construcción de conceptos y de conciencia ambiental. Claro está se debe continuar con este tipo de actividades y experiencias significativas que en últimas son las que van a quedar en nuestra construcción mental y que perduraran en el tiempo.

Estos mismos autores describen tres etapas o sistemas de referencia en su investigación que pueden ubicar los dibujos en función de la edad del individuo. Los estudiantes del Colegio Cristo Rey, tienen entre 9 y 12 años y están ubicados en un sistema de referencia coordinado o mapa de nivel 3 , y la condición requerida para el nivel 3 es que el dibujo debe incorporar todos los elementos ambientales hasta que la construcción contenga un posicionamiento e integración entre sí de relaciones que se dan dentro del ambiente.

Esto significa que se espera que los niños para sus edades ya tuvieran un nivel más claro del ambiente, sin embargo, con los primeros dibujos se evidencia una baja conceptualización y construcción de este concepto. En la segunda Fase ya se logró llegar a lo que se esperaba para estas edades. Con este resultado se puede confirmar que los niños tienen la capacidad por su edad de construir estas relaciones y conceptos, pero que se hace necesario que las estrategias utilizadas en el aula les den herramientas a los niños para elaborar estos conceptos tal como se hizo durante toda la jornada de interpretación ambiental. En este sentido, Ramírez (2006) y Rey (2009) plantearon que se debe permitir el desarrollo sensible y el uso de todos los sentidos en espacios libres sin barreras para ampliar el alcance de las opciones de exploración; para permitir que se dé el proceso de desarrollo sensorial. Los resultados muestran que su implementación ha dado lugar a nuevos aprendizajes y una actitud positiva hacia el desarrollo de actividades educativas relacionadas con el medio ambiente.

Por otro lado, se demuestra la generación de actitudes investigativas y el desarrollo de habilidades sensoriales y reflexivas de suma importancia y beneficiosas para el medio ambiente de la región. El uso de ejercicios y metáforas de desarrollo sensorial permite a los estudiantes ir más allá del simple conocimiento formal $y$ tocar el entorno real circundante, apoyando así las opiniones de Corral (1998) y Vega y Álvarez (2005) citadas por Rodríguez y Ramos (2008), cuando señalan que no basta solo con entender los temas ambientales, porque las acciones que se realizan en la práctica producirán mayores resultados; el uso de metáforas puede lograr resultados 
satisfactorios, confirmando así esto, las metáforas le dan estructura y significado a la realidad, que influye en la representación interna, la visión y la imagen del mundo por parte de la persona. Debido a estas metáforas, los estudiantes pueden expresar sus pensamientos y sentimientos sobre la responsabilidad, cuidado y protección de los recursos naturales de la zona de manera positiva.

\section{CONCLUSIONES}

La estrategia de interpretación ambiental que incluye un libreto metafórico y una serie de actividades sensoriales permite a los niños la construcción de conceptos ambientales que van a perdurar en el tiempo, lo que hace que sea una estrategia importante en los procesos de educación ambiental.

La metodología de relaciones ambientales nos permite tomar datos cualitativos y volverlos cuantitativos a pesar que cada dibujo es diferente $y$ es una representación de como los niños conciben su ambiente, para con ello establecer los avances obtenidos en la construcción de conocimiento.

Se debe seguir insistiendo en que la educación ambiental no es solo cosa de los docentes de ciencias naturales, - de campañas de recolección de residuos, sino que es una tarea más profunda en la que todos debemos estar inmersos docentes, padres de familia, instituciones del orden nacional, regional y local, las empresas privadas, la academia, las comunidades entre otros para realmente lograr los resultados esperados.

Este tipo de actividades de interpretación ambiental, que incluye la participación de todos los sentidos en espacios abiertos accesibles, permite generar la curiosidad de los niños por conocer y explorar, más allá de su conocimiento formal de aula.

Estas vivencias generan aprendizajes significativos que permiten permanecer en el tiempo, ya que son construcciones reales de conocimiento.

\section{REFERENCIAS}

Acosta, C., Fuenmayor, B., León, E., Sayago, A. (2006). Programa innovador de educación ambiental para la población indígena Añú en la laguna de Sinamaica. Revista Omnia, 12, (3): 123-150. Venezuela. Cánovas, C. (2002). Educación ambiental y cambio de valores en la sociedad. Observatorio medioambiental, 5, pp. 357-364.

Ángel Pérez, D. (2011). Hermenéutica y métodos de investigación de las Ciencias Sociales. Estudios de Filosofía, (44), 7. Recuperado el 6 de octubre de 2020, de http://www.scielo.org.co/scielo.php?sc ript $=$ sci arttext\&pid $=$ S012136282011000200002\&lng=en\&t|ng =e n.

Balcazar, F. (2003). Investigación acción participativa (iap):

Aspectos 
conceptuales y dificultades de implementación. Fundamentos en Humanidades, IV(7-8), 59-77. Disponible

en: https://www.redalyc.org/articulo.o a? id=184/18400804

Caballero,A (2002). Desarrollo de la presentación espacial, eduPsykhé, Vol. 1 , No. 1, 41-67

Gato,B (2012). ¿La entropía es contraria a la existencia de seres humanos?. El País. Recuperado de: https://elpais.com/elpais/2019/08/19/ ciencia/1566206604 137956.html

González, E. (2007). Educación ambiental. Trayectorias, rasgos y escenarios. México: Plaza y Valdés.

Goñi, A, Medrano, S., \& Palacios, S (2000). Conocimiento socio personal, conocimiento moral y valores. Revista de Psicodidáctica, (9),0. Disponible en: https://www.redalyc.org/articulo.oa?id $=175 / 17500902$

Holahan, C. (2005). Psicología ambiental, un enfoque general. México: Limusa Editores.

Molano, C. (2004). Desarrollo de la conciencia ambiental por medio de la lúdica, "Una propuesta desde la educación ambiental para el desarrollo rural". Revista Luna Azul, 18. Desde: http://lunazul.ucaldas.edu.co/index.ph p?option $=$ com content\&task =view\&id $=126 \&$ Itemid $=12$.

Novo, M. (2009). La educación ambiental, una genuina educación para el desarrollo sostenible. Universidad Nacional de Educación a distancia. Madrid (España). Revista de Educación, número extraordinario; pp. 195-217.

Sánchez, M. (2009). El juego y otras actividades lúdicas para la educación ambiental de los escolares. Revista Innovación y experiencias educativas, 14.

Stutzin, G. (1984) Un imperativo ecológico: reconocer los derechos de la naturaleza. En: Ambiente y Desarrollo. Vol. I. No 1. Universidad de Chile. Santiago. Chile, pp. 97-114.

Rodríguez, E. (2014). "Del derecho ambiental a los derechos de la naturaleza: sobre la necesidad del diálogo intercultural". Jurídicas. No. 1, Vol. 11, pp. 95-116. Manizales: Universidad de Caldas.

Tréllez, E. (2002). La educación ambiental comunitaria y la prospectiva: una alianza de futuro. Tópicos en educación ambiental, 4: pp. 7-21. 